\title{
Nutritimatson Metabolism
}

Acrylamide, food-related exposure 173

Acrylamide-contaminated food, questionnaire 173

Adipose tissue 54

Alcohol consumption 16

Alcoholic beverages 100

Alkaline phosphatase 107

Antioxidant activity 189

- status modulation 189

- supplementation 304

Apolipoproteins 397

Argan oil 196

Association studies 183

ATP-binding cassette transporter A1 397

Beta-glucan 141

Biological value, rabbits 407

Black tea 189

Blood glucose 64

- pressure 319

Body composition 71, 110

- fat, lactating mothers 110

- _ percentage 71

- mass index 3,16,83

Bone health 312

Breast feeding 83

- milk intake 110

Broadband ultrasound attenuation 312

Cadmium 296

Calcium oxalate stone formation, rats 132

- supplementation 132

Cardiovascular diseases 196

- risk 366

- - factors 100

Catechin(s) 54, 189

Cholesterol 9, 26, 100, 149, 366

- absorption 346

Coffee consumption 149

Copper 283

Cuminum cyminum 300

Cysteine 125

\section{D-Dimer 77}

Determinants, fruit/vegetable intake 228, 267

Diabetes 392

-, type-2 64

Dietary cholesterol 386

- fatty acids 304

- fibers 141

- intake 16

- proteins 312
Digestibility coefficient 407

Docosahexaenoic acid bioavailability 49

- - sources 325

- - supplementation 49

Environmental correlates, fruit/vegetable intake 255

EPIC Heidelberg study 16

Epigallocatechin gallate 54

Erythrocyte fatty acid composition 49

- plasma membrane 9

Ethanol 300, 319

Exercise 33, 125

Experimental lithiasis 132

Fasting, circadian variation 77

Fat-free mass 110

Fatty acid 304

- _ composition 300

Focus groups 228

Folic acid 125

Food consumption, diabetics 64

- frequency 236, 246

- habits 267

- lipids 196

Formula feeding 83

Free fatty acid 88

Fruit/vegetable intake 209, 212, 221, 228, 236, 246, 255, 267

Gene expression 54

Genomics 355

Glutathione 33

- peroxidase 149

- - activity, whole blood 118

Green tea 54

- - decoction 118

Head and neck cancer 95

Health and diet indicators 165

Hemoglobin adducts 173

Hepatic lipid peroxidation 304

- thiobarbituric acid-reactive substance 304

Hexadeuterated cholesterol 346

High-density lipoprotein 77, 397

HIV-infected children 373

HIV-negative children 373

Homocysteine 33, 77, 125, 149, 366

Honey 319

Hypophosphatasia 107

Immunonutrition 95

Inflammatory cytokine 149
Insulin resistance 381

Insulin-like growth factor-I 83

Intervention mapping protocol 267

Iron 118

Islamic fasting 77

Isoflavones 33

15-Isoprostane $F_{2 t} 149$

Isotope dilution 110

Lactation 110, 333

Lecithin:cholesterol acyltransferase 397

Lipid peroxidation 304

- profile 141, 283

Lipopolysaccharide 149

Lipoprotein(a) 42, 366

Lipoproteins 386

Liver steatosis 289

Malnutrition 373

Malondialdehyde 33

Membrane fluidity 392

Meriones shawi shawi 296

Metabolic syndrome 289

Metabolomics 355

Milk and oxidative damage 189

- content 325

- products, fat-modified 42

n-3 polyunsaturated fatty acids 386

n-6 polyunsaturated fatty acids 386

Net protein utilization and liver function 407

Nigerian citrus honey 319

Nutrition rehabilitation 373

Nutritional intervention study 196

Oat bran 141

Obesity 183, 381

-, rodents 54

-, Tehranian adults 3

-, severe 289

Olive oil 196

Omega-3 fatty acid 325

Overweight, premenopausal women 141

-, Tehranian adults 3

-, trends 3

Oxidative stress 283, 392

Peroxidizability index 304

_ _ value in rats 304

Phospholipase $\mathrm{A}_{2} 392$

Physical activity 183

- training 125

Phytosterols 346 
Plasma fatty acid composition 49

Polyunsaturated fatty acids 397

- /saturated fatty acid ratio 304

Postnatal development in mice 333

Precoded 24-hour recall, fruit/vegetable intake 236, 246

Prevalence of overweight and obesity 71

Pro Children study 209, 212, 221, 228, 236, 246, 255, 267

Protein efficiency ratio 407

- supplementation 132

Proteomics 355

Psychosocial correlates, fruit/vegetable intake 255

$\left[{ }^{14} \mathrm{C}\right]$ quercetin 178

Quercetin bioavailability 178

Quercetin-3-rutinoside 178

Ramadan, meal frequency 77

Rat small intestine 178

Ready-to-eat cereals 165

Resting energy expenditure 381

Retinoic acids 155

Retinoid concentrations 333

Retinol 155

Retinyl esters 155
Reverse cholesterol transport 397

Rutin 178

- bioavailability 178

School setting, fruit/vegetable promotion programme 267

Selenium 118

Smoking 16

Somatotropin 88

Spices 300

Spirulina (Spirulina platensis) 373

Staple foods 64

Sulphurous mineral water 9

Systems biology 355

TEAVIGO $^{\text {TM }} 54$

Tehran Lipid and Glucose Study 3

Thermally oxidized sunflower oil 300

Thiamine pyrophosphate 107

Thyroxine 88

Tissue accretion 325

Total antioxidant capacity 33

- - substances 283

- body water, lactating mothers

110

- protein, newborn lamb 88

Transcriptomics 355
Triacylglycerol 319

Tricipital skin-fold thickness 83

Triglycerides 26, 386

3,5,3'-Triiodothyronine 88

Uncoupling protein 3183

Uric acid 33

Urinary glucose 64

Validation, precoded questionaire 221

Vegan diet 366

Vegetable consumption 212, 221, 236, 255

Vitamin A 26

- - exposure 155

- _ safety 155

- - supplementation 333

- -, non-pregnant females 155

- $\quad$, single and repeated doses 155

- $\mathrm{B}_{6} 125$

- $\mathrm{B}_{12} 125$

- C 33

- E 26, 33, 392

Yemen, overweight/obesity 342

Zinc 118 